\title{
Design of a Full Stokes Polarimeter for Chromospheric Measurements with SOLIS/VSM
}

\author{
S. Gosain and J. W. Harvey \\ National Solar Observatory, 950 N Cherry Avenue, Tucson 85719, Arizona, USA \\ email: sgosain, jharvey@nso.edu
}

\begin{abstract}
The synoptic observations of the magnetic field of the Sun have continued at the National Solar Observatory (NSO) since 1970s. The daily full-disk maps of the longitudinal magnetic field are regularly combined to form Carrington maps of the photospheric magnetic flux per solar rotation. These maps continue to be used by the international research community for a variety of studies related to solar magnetism as well as for space weather studies. The current NSO synoptic facility is the Synoptic Optical Long-term Investigation of the Sun (SOLIS), which regularly provides photospheric vector and chromospheric longitudinal full-disk magnetograms, among other data products. In the near future, an upgrade of SOLIS to produce chromospheric vector magnetograms is planned. We present the design of a new polarization modulator package for full Stokes polarimetry of the chromospheric Ca II $854.2 \mathrm{~nm}$ spectral line.
\end{abstract}

Keywords. Sun: chromosphere; Instrumentation: polarimeters; Techniques: polarimetric

\section{Introduction}

The NSO Integrated Synoptic Program (NISP) provides long-term synoptic observations of the Sun to the international solar physics research community. One of the instrument systems operating under NISP is the SOLIS facility. SOLIS, an acronym for Synoptic Optical Long-term Investigations of the Sun, is a modern synoptic facility and is described in Keller (2000). SOLIS has a suite of instruments mounted on an equatorial mount (see Figure 1.), one of them is the Vector Spectro-Magnetograph (VSM) instrument. VSM is designed for the study of magnetic fields in the photosphere and the chromosphere over the full solar disk.

The VSM currently makes full-disk spectropolarimetric measurements in the photospheric Fe I $630.2 \mathrm{~nm}$ line pair and the chromospheric Ca II $854.2 \mathrm{~nm}$ line. VSM is a slit scanning spectrograph instrument. It uses a long slit to spatially scan the entire solar disk from pole-to-pole, while performing polarimetry at the aforementioned spectral lines at each slit-scan position. For photospheric lines VSM records the full Stokes vector $\mathrm{S}=[\mathrm{I}, \mathrm{Q}, \mathrm{U}, \mathrm{V}]^{T}$, while for the chromospheric line it currently records only the Stokes-I and $\mathrm{V}$ components. That means that in the chromosphere we are limited to longitudinal magnetic field measurements only. Currently VSM is being upgraded to perform full Stokes polarimetry in the chromospheric Ca II $854.2 \mathrm{~nm}$ spectral line, which will in principle allow the retrieval of the full magnetic field vector. To this end a new polarization modulator package is being developed at NSO. Preliminary optical design and the test results of the modulator are described here. 


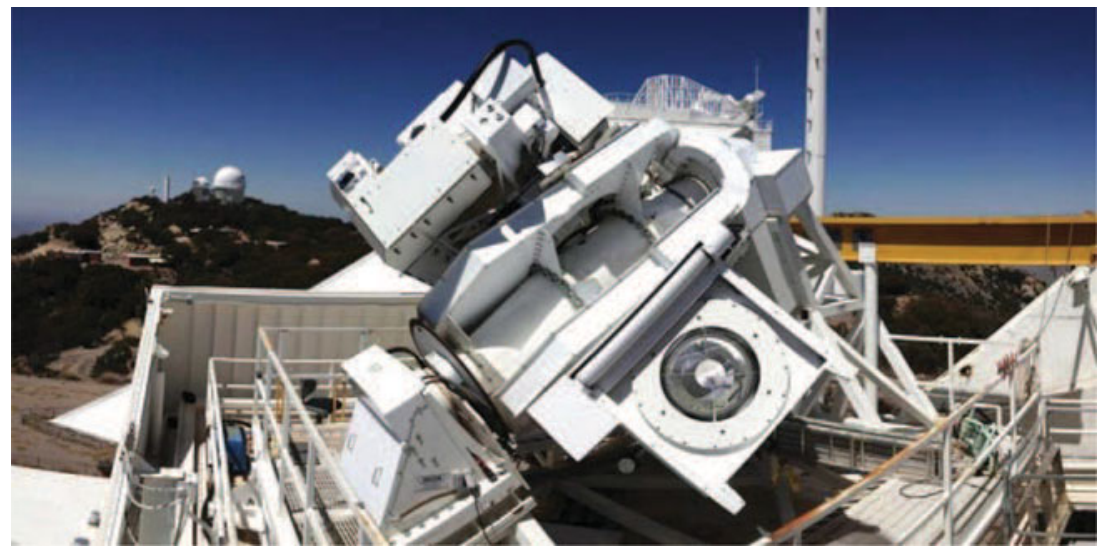

Figure 1. SOLIS Vector Spectro-Magnetograph (VSM).

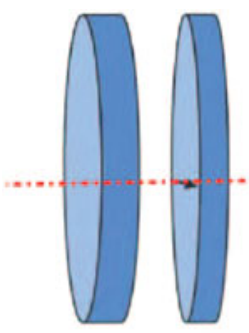

I. Filter FLC1

0 deg

$2.2 \mathrm{~mm}$

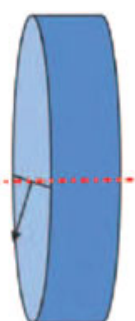

WP1

$246 \mathrm{deg}$

$10 \mathrm{~mm}$

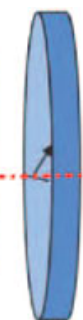

FLC2

$30 \mathrm{deg} 90 \mathrm{deg}$

$2.2 \mathrm{~mm} \quad 10 \mathrm{~mm}$

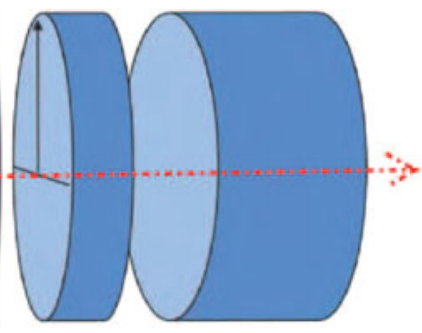

Glass Spacer

$19 \mathrm{~mm}$

Figure 2. Schematic of the modulator package construction. The orientation of polarizing optics and their thicknesses are given in the inset. FLC stands for Ferroelectric liquid crystal modulator and WP stands for fixed retardance polymer wave plate. Light enters from left side of the optical train.

\section{Motivation for the Chromospheric Magnetic Field Measurements}

The magnetic fields in the chromosphere play a dominant role in shaping its structure and dynamics (Harvey 2006). At this height the magnetic forces start to dominate over plasma pressure forces (Gary 2001). There are several reasons for which we want to study chromospheric vector fields, such as chromospheric heating (Hall 2008), coronal field extrapolations (Yelles Chaouche et al. 2012), flow of electric currents from the photosphere to the corona (Gosain et al. 2014), magnetic field configuration of filaments and flares (Harvey 2012), etc.

\section{Modulator Design}

The schematic design of the modulator package is shown in Figure 2. The first element is a narrow band interference filter (FWHM of $4 \mathrm{~nm}$; centered at $854.2 \mathrm{~nm}$ ). Following the pre-filter are a Ferroelectric Liquid Crystal (FLC), a fixed polymer retarder, another FLC, and another fixed polymer retarder. Finally, at the end of the optical train a glass spacer is required for the purpose of maintaining the correct focus on the camera. The interference filter helps to reject unwanted light in the spectrograph and also to protect FLCs from ultra-violet damage. 

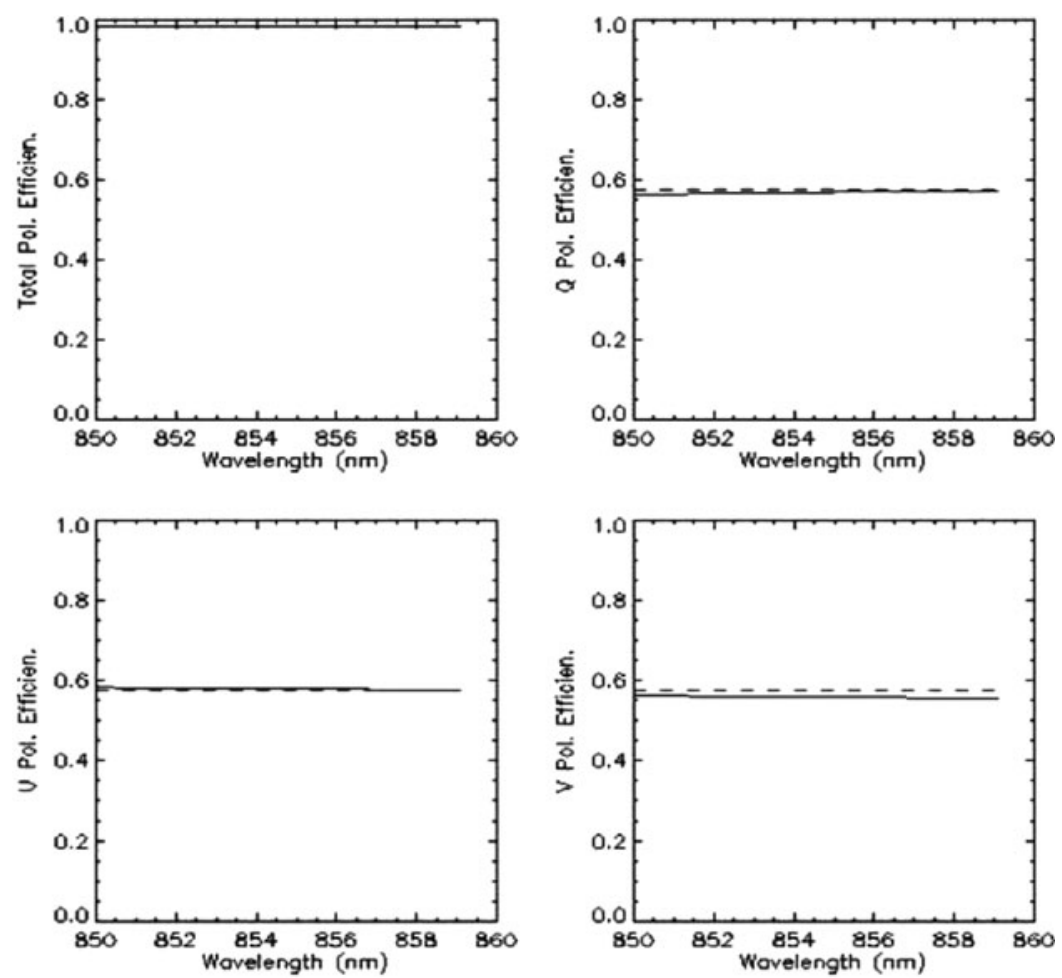

Figure 3. Dashed line shows maximum balanced demodulation efficiency that can be achieved for a four-state modulation scheme. Solid line shows the computed efficiency expected for the selected design parameters of the modulator.

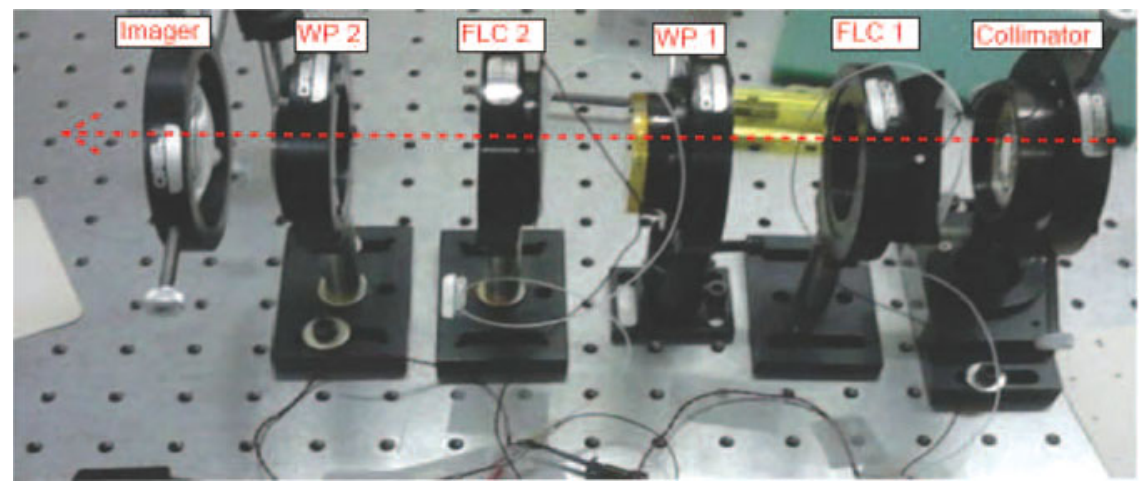

Figure 4. Laboratory setup for the testing of the modulator package. Light enters from the right side of the optical train.

The modulation scheme is a four-state balanced modulation scheme (Keller et al. 2003). The orientation of the various polarization optics components in the modulator package is optimized to achieve the best demodulation efficiency, $\eta_{D}$, for Stokes $\mathrm{Q}, \mathrm{U}$ and $\mathrm{V}$, following del Toro Iniesta (2000). The orientations that yield the optimum $\eta_{D}$, in the wavelength range $(850-860 \mathrm{~nm})$, were determined by using a software program that uses a genetic algorithm (Charbonneau 1995) to find the best solution in the parameter space. Figure 3 shows, for Stokes Q, U and V, the maximum achievable $\eta_{D}$ (dashed line) for a four state modulation scheme and the value expected for our design (solid line). 

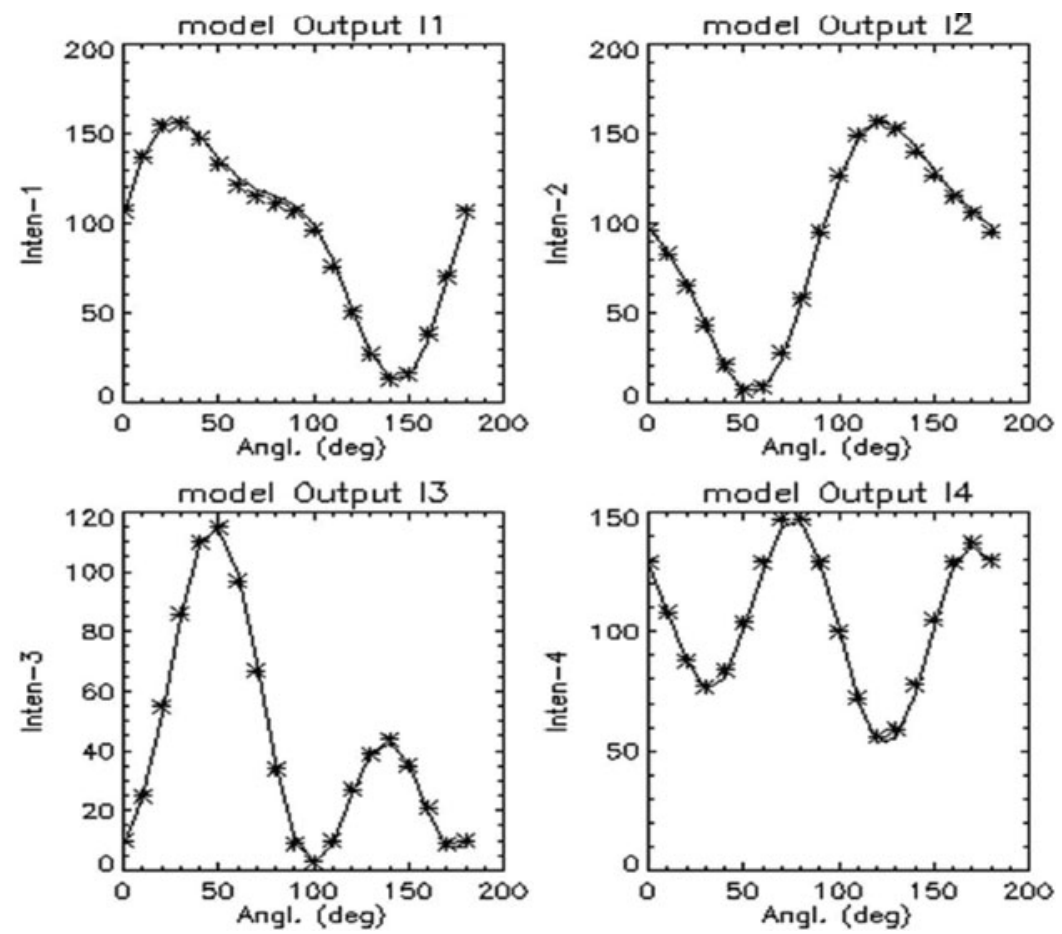

Figure 5. Measurement of the modulated intensity signal (asterisks) for various known input polarization vectors, and the computed value (solid line) for the design parameters of the modulator.

Figure 4 shows the laboratory test setup for determining the performance of the modulator package. We align the orientations of the optical components according to the design values (as depicted at the bottom of the schematic shown in Figure 2). The polarizing optics are mounted on rotatable mounts for alignment. We feed the modulator optics with a laboratory light source (854 nm light emitting diode). Various known input polarization states are produced by the combination of an input polarizer (horizontally oriented, $0^{\circ}$, right handed coordinate system) and a variable rotatable retarder (Soleil compensator). These immediately follow the light source. In particular, we fix the retardance of Soleil to $90^{\circ}$ at $854 \mathrm{~nm}$ and rotate it counterclockwise in ten degree angular steps (varying from 0 to $180^{\circ}$ ). A crossed polarizer in front of the photo-detector acts as the analyzer (vertically oriented, $90^{\circ}$ ). We then modulate in such a way that FLC1 is driven at $75 \mathrm{~Hz}$ and FLC2 at $150 \mathrm{~Hz}$. This gives rise to a four-state intensity modulation which is shown with asterisks in Figure 5, while the solid line depicts the computed modulation expected from our design parameters. A close agreement between the two verifies the efficiency of the design.

\section{References}

Charbonneau, P. 1995, ApJS 101, 309

Gary, G. A. 2001, Solar Phys. 203, 71

Gosain, S., Démoulin, P., \& López Fuentes, M. 2014, ApJ 793, 15

Hall, J. C. 2008, Living Reviews in Solar Physics 5, 2

Harvey, J. W. 2006, in: Casini, R. \& Lites, B. W. (eds), Solar Polarization IV ASP Conf. Series 358,419 
Harvey, J. W. 2012, Solar Phys. 280, 69

Keller, C. U. 2000, JA\&A 21, 127

Keller, C. U., Harvey, J. W., \& Giampapa, M. S. 2003, in: Keil, S. L. \& Avakyan, S. V. (eds)., Innovative Telescopes and Instrumentation for Solar Astrophysics, Proc. of SPIE 4853, 194

Keller, C. U., Solis Team 2001, in Sigwarth, M. (ed.), Advanced Solar Polarimetry - Theory, Observation, and Instrumentation - 20TH NSO/Sac Summer Workshop, ASP Conf. Series, 236,16

del Toro Iniesta, J. C. \& Collados, M. 2000, Applied Optics 39, 1637

Yelles Chaouche, L., Kuckein, C., Martínez Pillet, V., \& Moreno-Insertis, F. 2012, ApJ 748, 23 